\title{
Expression of PIM-1 in salivary gland adenoid cystic carcinoma: Association with tumor progression and patients' prognosis
}

\author{
XIN ZHU ${ }^{1}$, YUNFANG YU ${ }^{2}$, XIUXIU HOU ${ }^{2}$, JIAJIE XU ${ }^{2}$, ZHUO TAN $^{2}$, \\ XILIN NIE ${ }^{2}$, ZHIQIANG LING $^{1}$ and MINGHUA GE ${ }^{2}$ \\ ${ }^{1}$ Zhejiang Cancer Research Institute; ${ }^{2}$ Department of Head and Neck Surgery, \\ Zhejiang Cancer Hospital, Hangzhou, Zhejiang 310022, P.R. China
}

Received December 18, 2015; Accepted October 13, 2017

DOI: $10.3892 / \mathrm{ol} .2017 .7408$

\begin{abstract}
Pim-1 proto-oncogene, serine/threonine kinase (PIM-1) phosphorylates a series of substrates to exert its oncogenic function in numerous malignancies. The present study investigated the clinical significance of the PIM-1 protein, apoptosis status and apoptosis-associated proteins, including forkhead box O3a (FOXO3a), B cell lymphoma-2 (BCL-2) and BCL-2-associted agonist of cell death (BAD), were investigated in salivary gland adenoid cystic carcinoma (ACC) tissues. PIM-1 expression levels in 4 pairs of ACC tissues and corresponding normal salivary gland tissues were determined by western blot analysis. PIM-1, FOXO3a, BAD and BCL-2 expression levels in $60 \mathrm{ACC}$ tissues were evaluated by immunohistochemistry (IHC). A terminal deoxynucleotidyl-transferase-mediated dUTP nick end labeling assay was performed to detect the apoptosis status of ACC tissues. PIM-1 was revealed to be highly expressed in ACC tissues compared with adjacent normal tissues. IHC staining results demonstrated high expression ratios of PIM-1, FOXO3a, BCL-2 and BAD [33.33\% (20/60), 51.67\% (31/60), 51.67\% (31/60) and 55\% (33/60)], respectively, and significant correlations between the expression of PIM-1 and FOXO3a and BCL-2 $(\mathrm{P}<0.05)$. Apoptotic rates were significantly associated with PIM-1, FOXO3a, BCL-2 and BAD expression levels $(\mathrm{P}<0.05)$. PIM-1 expression levels were significantly associated with tumor size, lymph node involvement, nerve invasion, distant metastasis and weakly associated with tumor node metastasis stage. Kaplan-Meier survival curves revealed that PIM-1 expression level was significantly associated
\end{abstract}

Correspondence to: Professor Minghua Ge, Department of Head and Neck Surgery, Zhejiang Cancer Hospital, 38 Guangji Road, Hangzhou, Zhejiang 310022, P.R China

E-mail: gemh@zjcc.org.cn

Abbreviations: ACC, adenoid cystic carcinoma; IHC, immunohistochemistry; TUNEL, terminal deoxynucleotidyltransferase-mediated dUTP nick end labeling

Key words: salivary adenoid cystic carcinoma, PIM-1, apoptosis, prognosis with disease-free survival of patients with ACC ( $\mathrm{P}=0.009)$. Cox regression multivariate analysis results revealed that histotype, distant metastasis and apoptotic rate were independent prognosis factors for ACC. Assessment of PIM-1 may be useful in investigating the malignant behaviors of ACC and predicting the outcome of patients with ACC.

\section{Introduction}

Salivary gland adenoid cystic carcinoma (ACC) accounts for $\sim 10 \%$ of cases of epithelial salivary tumors and has a low 5 -year survival rate $(<20 \%$ in patients with highly metastatic tumors) $(1,2)$. The development of ACC involves the interaction of oncogenes and tumor suppressor genes, similar to most other types of tumor (3). However, the precise mechanisms underlying ACC carcinogenesis remain to be elucidated $(4,5)$.

PIM kinases are oncogenic and are known to phosphorylate numerous substrates to exert their functions and have important roles in numerous malignancies. PIM-1, Pim-1 proto-oncogene, serine/threonine kinase (PIM-1) is upregulated in a number of cancer subtypes and the overexpression of PIM-1 is thought to be involved in cancer-specific apoptosis signaling pathways (6-12). Apoptosis is modulated by complex pathways that involve a series of apoptosis-associated proteins. As a substrate of PIM-1 kinase, Forkhead box O3a (FOXO3a) is a proapototic transcription factor and regulates the expression of numerous apoptosis-associated genes to induce apoptosis (13). It has previously been revealed that the invalidation of FOXO3a by PIM-1 may downregulate its transcriptional function and aid cell survival (14). It has been previously established that the B cell lymphoma-2 (BCL-2) family includes the most well-known apoptosis-associated proteins (15). As an anti-apoptotic factor of the BCL-2 family, BCL-2 is able to restrain the mitochondrial permeability transformation and interact with proapoptotic proteins to inactivate them. BCL-2-associted agonist of cell death (BAD), a proapoptotic member of the BCL-2 family, normally binds to the BCL-2/BCL-X complex and triggers apoptosis. A previous study demonstrated that PIM-1 physically interacted with BAD and was suggested to be an essential molecular mechanism underlying PIM-1-modulated cell apoptosis (16). This evidence indicated that FOXO3a, BAD and BCL-2 were involved in the PIM-1-associated apoptosis process (13-16). 
The present study evaluated protein expression levels of PIM-1 and apoptosis-associated proteins, including FOXO3a, BAD and BCL-2 in 60 ACC tissues by immunohistochemistry (IHC). Terminal deoxynucleotidyl-transferase-mediated dUTP nick end labeling (TUNEL) assay was performed to evaluate the apoptosis rate in ACC tissues. The associations between PIM-1 and apoptotic status and apoptosis-associated proteins were deduced. Furthermore, associations between PIM-1, apoptotic status, apoptosis-associated proteins and clinical parameters, including prognosis, were analyzed.

\section{Materials and methods}

Tissue specimens. The present study was approved by the Ethics Committee of Zhejiang Cancer Hospital (Hangzhou, China). A total of 60 paraffin-embedded ACC tissue samples, 4 fresh ACC tissues and 4 fresh normal salivary gland tissues were obtained from Zhejiang Cancer Hospital between November 2002 and April 2013. These were taken from the hospital bank archive, and subsequently were 'freshly prepared' between the years 2002-2013, and so they were still covered by the retrospective approval form provided. There were 23 male, 37 female patients and the mean age was 51 (range between 28 and 78). All patients underwent surgical treatment and all tumor samples were histopathologically confirmed to be ACC.

Western blot analysis. ACC tissue samples were ground with Tissue Lyser-II (Qiagen GmbH, Hilden, Germany) and lysed with radioimmunoprecipitation assay lysis buffer (P10013B; Beyotime Institute of Biotechnology, Haimen, China). Subsequently, the BCA Protein Assay kit (Beyotime Institute of Biotechnology, Haimen, China) was used to evaluate the protein concentration. All samples were maintained at $-70^{\circ} \mathrm{C}$ prior to electrophoresis. Each sample containing $50 \mu \mathrm{g}$ of protein was separated using $12 \%$ SDS-PAGE. Following electrophoresis, the proteins were transferred from the gel to nitrocellulose membrane (Immobilon- $\mathrm{P}^{\mathrm{SQ}}$ Transfer Membrane; EMD Millipore, Billerica, MA, USA). Membranes were blocked in TBS buffer (50 mM Tris-Cl, $150 \mathrm{mM} \mathrm{NaCl}, \mathrm{pH}$ 7.6) supplemented with 5\% non-fat dry milk at room temperature for $3 \mathrm{~h}$. Subsequently, the membranes were incubated with primary antibodies against PIM-1 (EP2645Y; 1:1,000 dilution; Novus Biologicals, Ltd., Cambridge, UK) and GAPDH (R1208-3; 1:2,000 dilution; Huabio Technology, Hangzhou, China) at $4^{\circ} \mathrm{C}$ overnight prior to incubation with horseradish peroxidase-labeled Goat Anti-Rabbit IgG (G+L) (HA1001; 1:2,000 dilution; Huabio Technology) at room temperature for $3 \mathrm{~h}$. Subsequently, the membranes were washed in TBST (B1009; Applygen, Beijing, China) and exposed to $2 \mathrm{ml}$ enhanced chemiluminesence reagent (TJWBKLS0100; Tiengene, Guangzhou, China). The images were captured and analyzed using Bio-Rad GelDoc XR (Image Lab 4.1; Bio-Rad Laboratories, Inc., Hercules, CA, USA).

$I H C$. Sections (4- $\mu$ m thick) of paraffin-embedded tissues were cut, mounted on glass slides (MS-coated glass; Matsunami Glass Ind., Ltd., Kishiwada, Japan) and dried overnight at $37^{\circ} \mathrm{C}$. Following deparaffinization and antigen retrieval in $0.01 \mathrm{M}$ citrate buffer, the slides were inactivated for endogenous peroxidase activity in $3 \% \mathrm{H}_{2} \mathrm{O}_{2}$ /methanol and incubated

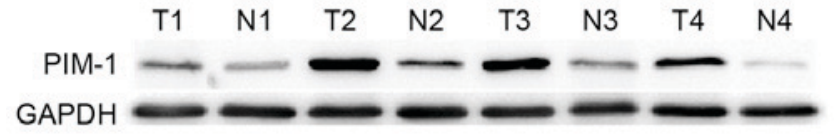

Figure 1. Western blot analysis was performed to analyze the expression levels of PIM-1 protein in 4 pairs of adenoid cystic carcinoma tissues. The expression level of GAPDH was used as a quantitative control. PIM-1, Pim-1 proto-oncogene, serine/threonine kinase; T, adenoid cystic carcinoma tumor tissue; $\mathrm{N}$, corresponding normal salivary gland tissue.

with antibodies for PIM-1 (EP2645Y; 1:200 dilution; Novus Biologicals, Ltd.), FOXO3a (10849-1-AP; 1:200 dilution; ProteinTech Group, Inc., Chicago, IL, USA), BCL-2 (15071; 1:400 dilution; Cell Signaling Technology, Inc., Danvers, MA, USA) or BAD (ab32445; 1:200 dilution; Abcam, Cambridge, UK) at $4^{\circ} \mathrm{C}$ overnight. The streptavidin-biotin peroxidase staining kit (Histofine Simple Stain Max PO Multi; Nichirei, Tokyo, Japan) and DAB solution (Simple Stain DAB; Nichirei) were used to detect immunoreactivity. Images were captured under a light microscope at x200 magnification.

Evaluation of IHC staining. The staining results of IHC for no staining, light yellow, yellow-brown and brown were defined as the mean of $0,1,2$ and 3 staining intensity score, respectively. The staining distribution scores were presented as the percentage of nuclei staining positive cells and the total cells as $0,1-25,26-50,51-75$ and $>75 \%$ and defined as $0,1,2$, 3 and 4, respectively (17). The criterion of final scores was evaluated by multiplying the staining intensity score and the staining distribution score. Those with a score of $<5$ were defined as 'low expression' and those with a score of $\geq 5$ were considered as 'high expression'.

TUNEL assay. TUNEL staining was performed to quantify apoptosis. Sections ( $4-\mu \mathrm{m}$ thick) of paraffin-embedded ACC tissues were cut and stained with the TUNEL kit (Roche Applied Science, Madison, WI, USA), according to the manufacturer's instructions. Briefly, tissue sections were incubated with proteinase $\mathrm{K}$ for $20 \mathrm{~min}$ at $37^{\circ} \mathrm{C}$, and rinsed twice with PBS (5 min each). Subsequently, TUNEL reaction mixture (terminal deoxynucleotidyl transferase (TdT) buffer: TdT end-labeling cocktail $=1: 9$ ) was added to the samples at $37^{\circ} \mathrm{C}$ for $60 \mathrm{~min}$ and washed with PBS. Next, sections were treated with converter POD at $37^{\circ} \mathrm{C}$ for 30 min and colored with DAB.

Cancer cells with dark brown nuclei were considered apoptotic when investigated under a light microscope at x200 magnification. The TUNEL index for ACC tissues was evaluated by the percentage of positive cells in one field of vision. At least three fields were randomly selected and 200 cells in each area were counted per slide.

Statistical analysis. SPSS version 10.0 (SPSS, Inc., Chicago, IL, USA) was used to analyze all experimental data. The data are presented as 'high' and 'low' levels. Associations between PIM-1 and FOXO3a, BCL-2, BAD expression levels, apoptotic rate and the clinical parameters in ACC tissues were analyzed using the $\chi^{2}$ test or Fisher's exact test. The Kaplan-Meier method was used to perform survival analysis and significant differences were evaluated by means of the log-rank test. Multivariate analysis with the Cox regression model was used 
A

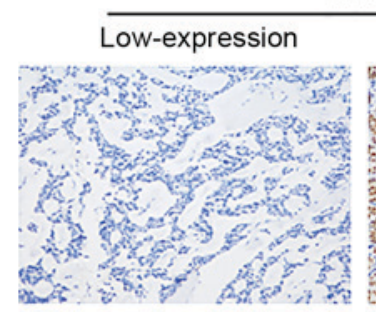

C

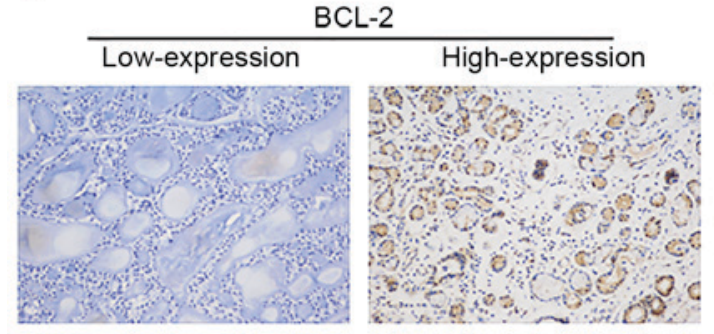

$\mathrm{E}$
PIM-1

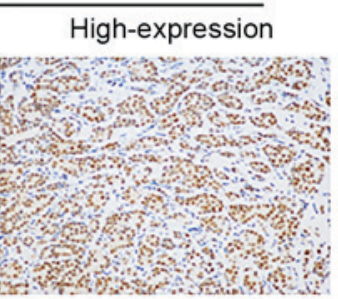

$B C L-2$

Apoptotic rate

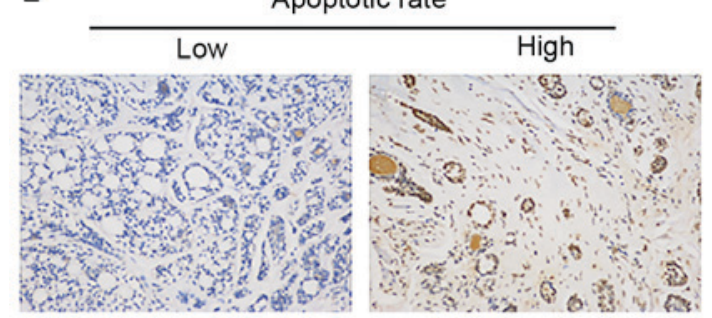

B

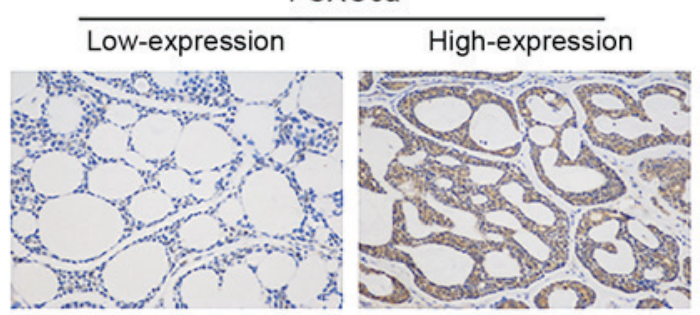

D

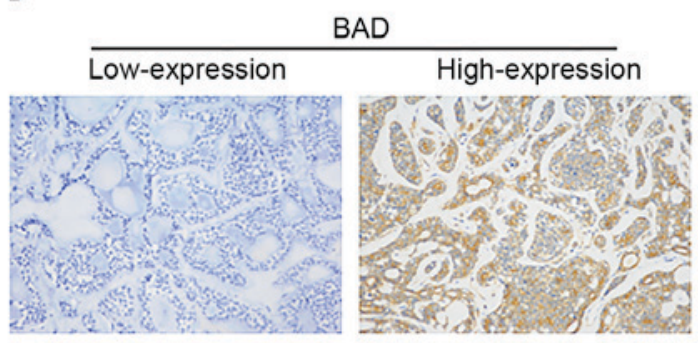

Figure 2. Immunohistochemical staining and terminal deoxynucleotidyl-transferase-mediated dUTP nick end labeling staining results in ACC tissues. Expression levels of (A) PIM-1, (B) FOXO3a, (C) BCL-2 and (D) BAD in ACC tissues. (E) Low apoptotic rate and high apoptotic rate images. The nuclei of apoptotic cells are stained brown. Magnification of all images, x200. ACC, adenoid cystic carcinoma; FOXO3a, forkhead box O3a; BCL-2, B cell lymphoma-2; BAD, BCL-2-associted agonist of cell death

to determine the prognostic factors. $\mathrm{P}<0.05$ was considered to indicate a statistically significant difference.

\section{Results}

Protein expression levels of PIM-1 in ACC tissues and corresponding normal salivary gland tissues. A total of 4 matched pairs of ACC tissue and corresponding normal salivary gland tissue were randomly selected for western blot analysis. As presented in Fig. 1, PIM-1 protein expression was higher in tumor tissues compared with in matched normal tissues.

Expression levels of PIM-1, FOXO3a, BCL-2 and BAD in ACC tissues. IHC staining for protein expression levels of PIM-1, FOXO3a, BCL-2 and BAD in ACC tissues are shown in Fig. 2. High expression ratios of PIM-1, FOXO3a, BCL-2 and BAD were $33.33 \%$ (20/60), 51.67\% (31/60), 51.67\% (31/60) and 55\% (33/60), respectively. Table I presents significant associations between the expression of PIM-1 and FOXO3a $(\mathrm{P}=0.018)$. Furthermore, the expression of BCL-2 also had a significant association with the expression of PIM-1 $(\mathrm{P}=0.044)$. There was no association between the expression of PIM-1 and BAD.

TUNEL staining indicates apoptosis status of ACC tissues. The apoptotic rate analyzed by a TUNEL assay in ACC tissues was $36.65 \pm 23.94$ (range from 5 to 94 ). Those with a score $<36.65$ were defined as 'low apoptotic rate' and those with a score $\geq 36.65$ were considered to have a 'high apoptotic rate'. Fig. 2E presents the TUNEL assay stain results of low/high apoptotic rate tissues. As presented in Table II, the apoptotic rate was significantly associated with PIM-1, FOXO3a, BCL-2 and BAD expression levels. Patients with higher apoptotic rates tended to have lower PIM-1 and BCL-2 expression levels, and higher FOXO3a and BAD expression levels. Furthermore, the present study investigated the associations between apoptotic rate and clinical parameters, including sex, age, T-status, tumor node metastasis (TNM) stage, tumor location, tumor size, histological type, lymph node involvement, nerve invasion and distant metastasis. However, apoptotic rate had no significant associations with any clinical index.

Association between the expression of PIM-1, FOXO3a, $B C L-2$ and BAD levels, and the clinical characteristics in ACC tissues. As presented in Table III, PIM-1 levels were significantly associated with tumor size, lymph node involvement, nerve invasion and distant metastasis, whereas theywere weakly associated with TNM stage. FOXO3a expression level was closely associated with T-status, tumor size and lymph node involvement. There were significant associations between BAD level and TNM stage and distant metastasis. BCL-2 expression level revealed no significant associations with any clinical index.

Survival analysis. In the present study, patients' average follow-up time was $68.23 \pm 38.69$ months (mean \pm standard 
Table I. Association between the expression levels of PIM-1 and FOXO3a and BCL-2 and BAD in ACC tissues.

\begin{tabular}{|c|c|c|c|c|c|}
\hline & \multirow[b]{2}{*}{ Variables } & \multicolumn{2}{|c|}{ PIM-1 expression } & \multirow[b]{2}{*}{ Kappa value } & \multirow[b]{2}{*}{ P-value } \\
\hline & & Low 40 & High 20 & & \\
\hline \multicolumn{6}{|c|}{ FOXO3a } \\
\hline Low & 29 & 15 & 14 & -0.286 & $0.018^{\mathrm{a}}$ \\
\hline High & 31 & 25 & 6 & & \\
\hline \multicolumn{6}{|l|}{ BCL-2 } \\
\hline Low & 29 & 23 & 6 & 0.242 & $0.044^{\mathrm{a}}$ \\
\hline High & 31 & 17 & 14 & & \\
\hline \multicolumn{6}{|l|}{ BAD } \\
\hline Low & 27 & 15 & 12 & -0.194 & 0.099 \\
\hline High & 33 & 25 & 8 & & \\
\hline
\end{tabular}

${ }^{\mathrm{a}} \mathrm{P}<0.05$ by Kappa analysis. FOXO3a, forkhead box O3a; BCL-2, B cell lymphoma-2; BAD, BCL-2-associted agonist of cell death; ACC, adenoid cystic carcinoma; PIM-1, Pim-1 proto-oncogene, serine/threonine kinase.

Table II. Association between the apoptotic rate and PIM-1, FOXO3a, BCL-2 and BAD expression levels in ACC tissues.

\begin{tabular}{|c|c|c|c|c|c|}
\hline & \multirow[b]{2}{*}{ Variables } & \multicolumn{2}{|c|}{ Apoptotic rate } & \multirow[b]{2}{*}{ Kappa value } & \multirow[b]{2}{*}{ P-value } \\
\hline & & Low 34 & High 26 & & \\
\hline \multicolumn{6}{|l|}{ PIM-1 } \\
\hline Low & 40 & 17 & 23 & -0.395 & $0.002^{\mathrm{a}}$ \\
\hline High & 20 & 17 & 3 & & \\
\hline \multicolumn{6}{|c|}{ FOXO3a } \\
\hline Low & 29 & 21 & 8 & 0.303 & $0.017^{\mathrm{a}}$ \\
\hline High & 31 & 13 & 18 & & \\
\hline \multicolumn{6}{|l|}{ BCL-2 } \\
\hline Low & 29 & 12 & 17 & -0.294 & $0.021^{\mathrm{a}}$ \\
\hline High & 31 & 22 & 9 & & \\
\hline \multicolumn{6}{|l|}{ BAD } \\
\hline Low & 27 & 20 & 7 & 0.309 & $0.014^{\mathrm{a}}$ \\
\hline High & 33 & 14 & 19 & & \\
\hline
\end{tabular}

${ }^{\mathrm{a}} \mathrm{P}<0.05$ by Kappa analysis. FOXO3a, forkhead box O3a; BCL-2, B cell lymphoma-2; BAD, BCL-2-associted agonist of cell death; ACC, adenoid cystic carcinoma; PIM-1, Pim-1 proto-oncogene, serine/threonine kinase.

deviation; range from 15 to 156 months). Patients with a follow-up of $>5$ years accounted for $36.7 \%$ of all patients. At the end of the follow-up, 4 patients $(6.7 \%)$ were not included as data was not available, 10 patients (16.7\%) experienced disease recurrence, 20 patients $(33.3 \%)$ had passed away and 36 patients $(60 \%)$ remained alive.

Kaplan-Meier survival curves (Fig. 3A) demonstrated that the PIM-1 level was weakly associated with the overall survival of patients with ACC $(\mathrm{P}=0.062)$. patients with higher PIM-1 expression levels had a poorer prognosis compared with patients with lower PIM-1 expression levels. Fig. 3B revealed that apoptotic rate, reflected by TUNEL results, had a significant impact on the prognosis of patients $(\mathrm{P}=0.033)$. The outcomes for patients with higher apoptotic rates were more favorable compared with those with lower apoptotic rates. Additionally, the present study revealed that there was a significant association between tumor histotype and the survival of patients with ACC ( $\mathrm{P}=0.038$; Fig. 3C). However, other clinicopathological parameters, including sex, age, T-status, TNM stage, tumor location, tumor size, lymph node involvement, nerve invasion, distant metastasis and other proteins, including FOXO3a, BCL-2 and BAD levels, had no significant impact on the overall survival of patients with ACC. Furthermore, the present study demonstrated that PIM-1 expression level was significantly associated with disease-free survival of patients with ACC ( $\mathrm{P}=0.009$; Fig. 3D). It is worth noting that Cox regression multivariate analysis revealed that histotype, distant metastasis and apoptotic rate were independent prognosis factors for patients with ACC $(\mathrm{P}<0.05$; Table IV). 


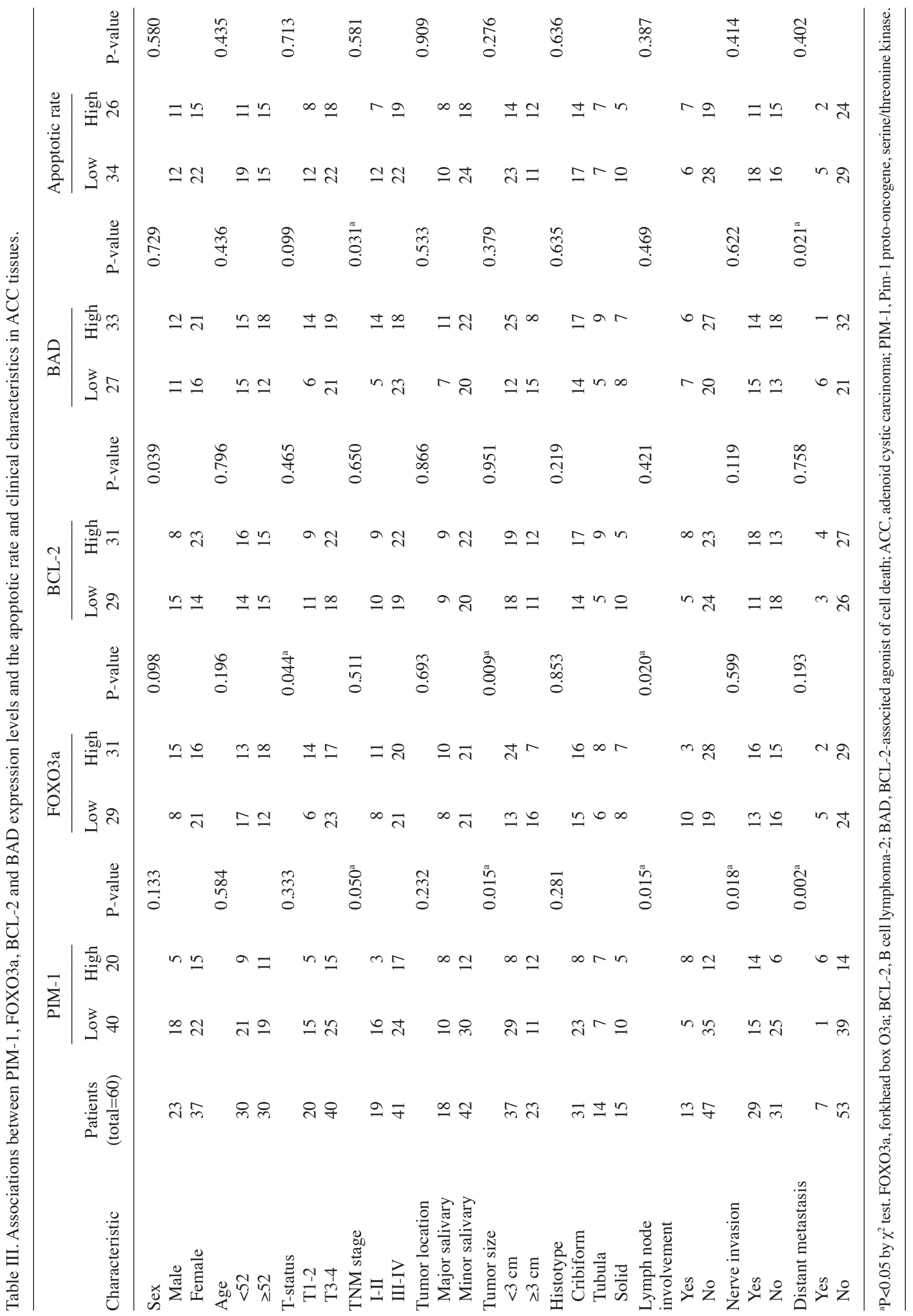


A

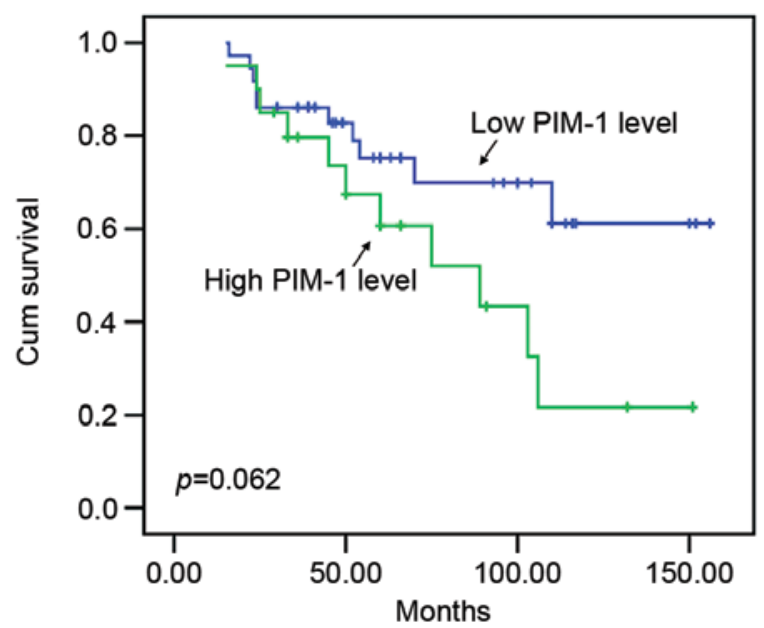

C

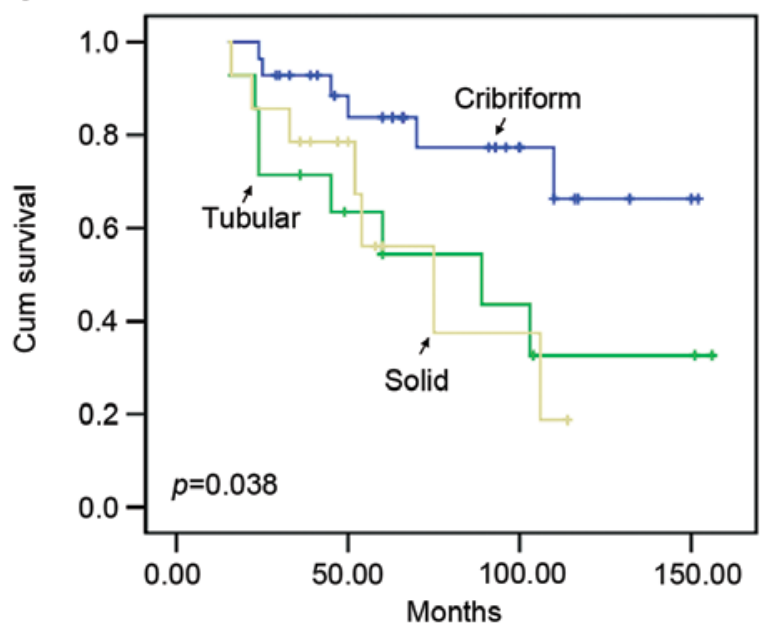

B

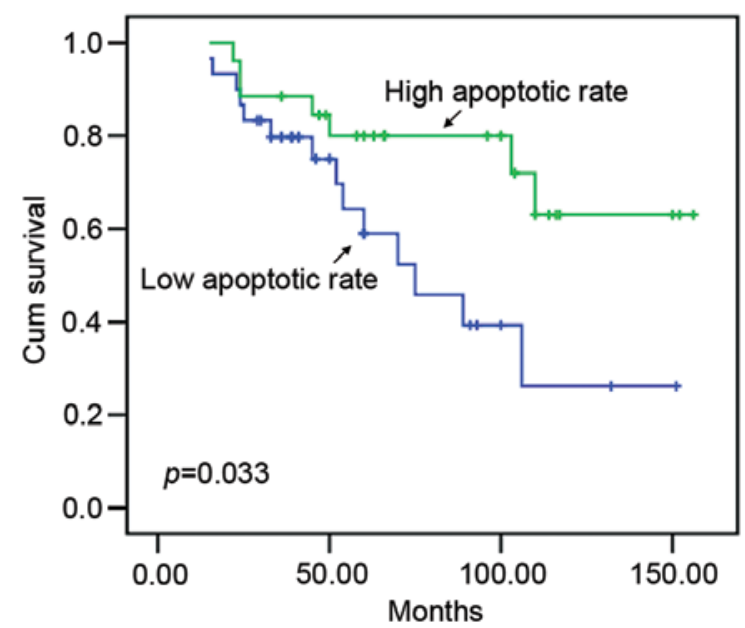

D

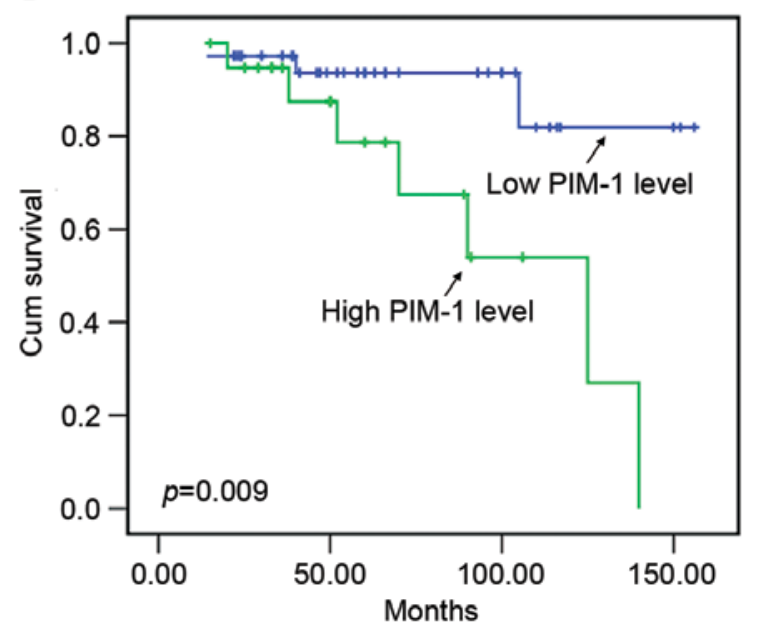

Figure 3. Kaplan-Meier curve analysis. (A) Association between PIM-1 protein expression level, which was analyzed by immunohistochemistry, and survival time of patients with ACC. (B) Association between apoptotic rate, analyzed by terminal deoxynucleotidyl-transferase-mediated dUTP nick end labeling assay, and survival time of patients with ACC. (C) Association between tumor histotype and survival time of patients with ACC. (D) Association between PIM-1 protein expression determined using immunohistochemistry and disease-free survival time of ACC patients. P-values were calculated using the log-rank test. ACC, adenoid cystic carcinoma; Cum, cumulative; PIM-1, Pim-1 proto-oncogene, serine/threonine kinase.

\section{Discussion}

Previous studies have suggested that PIM-1 is important for carcinogenesis and metastasis in numerous types of human cancer (6-12). The present study selected 4 pairs of ACC tissue and corresponding normal tissues and revealed that PIM-1 was overexpressed in ACC. IHC results demonstrated that PIM-1 was highly expressed in $33.33 \%$ (20/60) of ACC tissues. Furthermore, PIM-1 expression was significantly associated with tumor size, lymph node involvement, nerve invasion and distant metastasis. Patients with larger, lymph node involvement positive, nerve invasion positive and distant metastasis positive ACC tissues revealed higher PIM-1 levels. There was a weak association between the expression level of PIM-1 and ACC TNM stage. Survival analysis demonstrated that patients with higher PIM-1 levels had a shorter disease-free survival time and poorer prognosis. This suggested that upregulation of PIM-1 may promote the disruption of cell proliferation and homeostasis to drive malignant aggression and lead to a poor outcome.

Dysregulation of apoptosis is a vital feature of oncogenes and a number of previous studies have revealed that PIM-1 has an important role in apoptosis (18-20). The present study demonstrated that the apoptotic rate had a significant association with PIM-1 expression level in ACC patients' tissues. Patients with higher PIM-1 levels tended to have lower apoptotic rates. These results suggested the importance of PIM-1 in tumorigenesis of ACC, which involved the imbalance of apoptotic events. The associations between apoptosis, degree of malignancy and the prognosis of cancer patients have been well-studied (21-26). Patients with high apoptotic rates have a more favorable outcome compared with those with low apoptotic rates (21-26). In the present study, the apoptotic rate analyzed by TUNEL assay was associated with a significant impact on the patients' prognosis and was revealed to be an independent prognostic factor. These results are consistent with previous studies and demonstrated the importance of apoptosis in the prediction of outcome in ACC (21-26).

As a proapototic transcription factor, FOXO3a is a phosphorylation substrate of PIM-1. FOXO3a may upregulate proapototic proteins, including Bim and FAS ligand to trigger apoptosis (13). The present study revealed that PIM-1 protein expression level had an inverse association with FOXO3a 
Table IV. Multivariate survival analysis of clinicopathologic data of the adenoid cystic carcinoma patients (Cox regression hazards model).

\begin{tabular}{lcrc}
\hline & \multicolumn{3}{c}{ Multivariate analysis } \\
\cline { 2 - 3 } & $95 \%$ CI for Exp(B) \\
\cline { 2 - 3 } Variable & Lower & Higher & P-value \\
\hline Sex & 0.529 & 6.074 & 0.348 \\
Age & 0.385 & 5.051 & 0.613 \\
T-status & 0.173 & 9.338 & 0.812 \\
TNM stage & 0.055 & 2.668 & 0.333 \\
Tumor location & 0.388 & 6.233 & 0.533 \\
Tumor size & 0.134 & 1.694 & 0.252 \\
Histotype & 0.056 & 0.778 & $0.020^{\mathrm{a}}$ \\
Lymph node involvement & 0.110 & 2.073 & 0.324 \\
Nerve invasion & 0.671 & 10.995 & 0.161 \\
Distant metastasis & 1.348 & 81.307 & $0.025^{\mathrm{a}}$ \\
PIM-1 & 0.319 & 5.055 & 0.734 \\
FOXO3a & 0.090 & 1.302 & 0.116 \\
BCL-2 & 0.346 & 3.220 & 0.923 \\
BAD & 0.550 & 5.614 & 0.342 \\
Apoptotic rate & 1.787 & 57.428 & $0.009^{\mathrm{a}}$ \\
\hline
\end{tabular}

${ }^{\mathrm{a}} \mathrm{P}<0.05$, by multivariate analysis. $\mathrm{CI}$, confidence interval; TNM, tumor node metastasis; FOXO3a, forkhead box O3a; BCL-2, B cell lymphoma-2; BAD, BCL-2-associted agonist of cell death.

expression level in ACC tissues. The significant association between FOXO3a level and apoptosis was observed in the present study. Furthermore, IHC results demonstrated that FOXO3a expression levels were closely associated with clinical parameters, including TNM stage, tumor size and lymph node involvement. Previous studies revealed that FOXO3a was significantly associated with clinical stage and lymph node involvement in nasopharyngeal carcinoma and ovarian cancer, which were in agreement with our current findings $(27,28)$.

The findings obtained from the ACC tissues in the present study also demonstrated that BCL-2 protein expression levels had a significant association with PIM-1 expression level, whereas BAD protein expression level had no association with PIM-1 level. Furthermore, the present study revealed that BAD protein expression level was significantly associated with TNM stage and distant metastasis. Taken together, these results suggest PIM-1 may exert its oncogenic function by regulating apoptosis, which involves the interaction of $\mathrm{BCL}-2$ family and FOXO3a proteins.

As a frequently occurring malignant epithelial neoplasm, ACC originates from the salivary glands $(1,2)$. The growth modes of ACCs are histologically categorized into three types: Cribriform, tubular and solid $(3,4)$. It has been previously established that solid types of tumor are markedly more malignant compared with the other two types (29). The present study did not observe associations between histotype and PIM-1, FOXO3a, BCL-2, BAD levels or apoptotic rate. However, the survival analysis demonstrated that tumor histotype was significantly associated with patient prognosis. Furthermore, Cox regression results revealed that histotype was an independent prognosis factor. The results of the present study were in line with previous studies, that suggested histotypes have important roles in the aggressive behavior of ACC $(30,31)$.

The results of the present study confirmed that PIM-1 was overexpressed in ACC tissues and associated with FOXO3a, BCL-2 expression and apoptotic rate. PIM-1 was revealed to be significantly associated with tumor size, lymph node involvement, nerve invasion and distant metastasis, and is weakly associated with patient survival. Furthermore, the present study determined that apoptotic rates were significantly associated with PIM-1, FOXO3a, BCL-2 and BAD expression levels in ACC tissues. The results of the present study also revealed that histotype, distant metastasis and apoptotic rate were independent prognosis factors. Considered together, the current findings suggested that PIM-1 kinase is a novel molecular biomarker and a promising prognostic marker for ACC.

\section{Acknowledgements}

The present study was supported by National Natural Science Foundation of China (grant no. 81202127), the Subproject of National High Technology Research and Development Program of China (grant no. 2014AA022402) and Zhejiang Province Natural Science Foundation (grant no. LY14H160014).

\section{References}

1. International Head and Neck Scientific Group: Cervical lymph node metastasis in adenoid cystic carcinoma of the sinonasal tract, nasopharynx, lacrimal glands and external auditory canal: A collective international review. J Laryngol Otol 130: 1093-1097, 2016.

2. van der Wal JE, Becking AG, Snow GB and van der Waal I: Distant metastases of adenoid cystic carcinoma of the salivary glands and the value of diagnostic examinations during follow-up. Head Neck 24: 779-783, 2002.

3. Gondivkar SM, Gadbail AR, Chole R and Parikh RV: Adenoid cystic carcinoma: A rare clinical entity and literature review. Oral Oncol 47: 231-236, 2011.

4. Liu J, Shao C, Tan ML, Mu D, Ferris RL and Ha PK: Molecular biology of adenoid cystic carcinoma. Head Neck 34: 1665-1677, 2012.

5. Moskaluk CA: Adenoid cystic carcinoma: Clinical and molecular features. Head Neck Pathol 7: 17-22, 2013.

6. Alvarado Y, Giles FJ and Swords RT: The PIM kinases in hematological cancers. Expert Rev Hematol 5: 81-96, 2012.

7. Yan B, Yau EX, Samanta S, Ong CW, Yong KJ, Ng LK, Bhattacharya B, Lim KH, Soong R, Yeoh KG, et al: Clinical and therapeutic relevance of PIM1 kinase in gastric cancer. Gastric Cancer 15: 188-197, 2012.

8. Kim J, Roh M and Abdulkadir SA: Pim1 promotes human prostate cancer cell tumorigenicity and c-MYC transcriptional activity. BMC Cancer 10: 248, 2010.

9. Li S, Xi Y, Zhang H, Wang Y, Wang X, Liu H and Chen K: A pivotal role for Pim-1 kinase in esophageal squamous cell carcinoma involving cell apoptosis induced by reducing Akt phosphorylation. Oncol Rep 24: 997-1004, 2010.

10. Malinen M, Jääskeläinen T, Pelkonen M, Heikkinen S, Väisänen S, Kosma VM, Nieminen K, Mannermaa A and Palvimo JJ: Proto-oncogene PIM-1 is a novel estrogen receptor target associating with high grade breast tumors. Mol Cell Endocrinol 365: 270-276, 2013.

11. Jin Y, Tong DY, Tang LY, Chen JN, Zhou J, Feng ZY and Shao CK: Expressions of osteopontin (OPN), $\alpha v \beta 3$ and Pim-1 associated with poor prognosis in Non-small cell lung cancer (NSCLC). Chin J Cancer Res 24: 103-108, 2012. 
12. Weirauch U, Beckmann N, Thomas M, Grünweller A, Huber K, Bracher F, Hartmann RK and Aigner A: Functional role and therapeutic potential of the pim-1 kinase in colon carcinoma. Neoplasia 15: 783-794, 2013.

13. Nawijn MC, Alendar A and Berns A: For better or for worse: The role of Pim oncogenes in tumorigenesis. Nat Rev Cance 11: 23-34, 2011.

14. Morishita D, Katayama R, Sekimizu K, Tsuruo T and Fujita N: Pim kinases promote cell cycle progression by phosphorylating and down-regulating $\mathrm{p} 27 \mathrm{Kip} 1$ at the transcriptional and posttranscriptional levels. Cancer Res 68: 5076-5085, 2008.

15. Siddiqui WA, Ahad A and Ahsan H: The mystery of BCL2 family: Bcl-2 proteins and apoptosis: An update. Arch Toxicol 89: 289-317, 2015.

16. Magnuson NS, Wang Z, Ding G and Reeves R: Why target PIM1 for cancer diagnosis and treatment? Future Oncol 6: 1461-1478, 2010.

17. Ling ZQ, Guo W, Lu XX, Zhu X, Hong LL, Wang Z, Wang Z and Chen Y: A Golgi-specific protein PAQR3 is closely associated with the progression, metastasis and prognosis of human gastric cancers. Ann Oncol 25: 1363-1372, 2014.

18. Nensa F, Stattaus J, Morgan B, Horsfield MA, Soria JC, Besse B, et al: Dynamic contrast-enhanced MRI parameters as biomarkers for the effect of vatalanib in patients with non-small-cell lung cancer PIM kinases: An overview in tumors and recent advances in pancreatic cancer PIM kinases: An overview in tumors and recent advances in pancreatic cancer. Future Oncol 10: 823-833, 2014.

19. Xu J, Xiong G, Cao Z, Huang H, Wang T, You L, Zhou L, Zheng L, Hu Y, Zhang T and Zhao Y: PIM-1 contributes to the malignancy of pancreatic cancer and displays diagnostic and prognostic value. J Exp Clin Cancer Res 35: 133, 2016.

20. Matou-Nasri S, Rabhan Z, Al-Baijan H, Al-Eidi H, Yahya WB, Al Abdulrahman A, Almobadel N, Alsubeai M, Al Ghamdi S, Alaskar A, et al: CD95-mediated apoptosis in Burkitt's lymphoma B-cells is associated with Pim-1 down-regulation. Biochim Biophys Acta 1863: 239-252, 2017.

21. Wu X, Cai ZD, Lou LM and Zhu YB: Expressions of p53, c-MYC BCL-2 and apoptotic index in human osteosarcoma and their correlations with prognosis of patients. Cancer Epidemiol 36: 212-216, 2012.
22. Jia Y, Dong B, Tang L, Liu Y, Du H, Yuan P, Wu A and Ji J: Apoptosis index correlates with chemotherapy efficacy and predicts the survival of patients with gastric cancer. Tumour Biol 33: 1151-1158, 2012.

23. Adell GC, Zhang H, Evertsson S, Sun XF, Stål OH and Nordenskjöld BA: Apoptosis in rectal carcinoma: Prognosis and recurrence after preoperative radiotherapy. Cancer 91: 1870-1875, 2001

24. Fu DR, Kato D, Watabe A, Endo Y and Kadosawa T: Prognostic utility of apoptosis index, Ki-67 and survivin expression in dogs with nasal carcinoma treated with orthovoltage radiation therapy. J Vet Med Sci 76: 1505-1512, 2014.

25. Reséndiz-Martínez J, Asbun-Bojalil J, Huerta-Yepez S and Vega M: Correlation of the expression of YY1 and Fas cell surface death receptor with apoptosis of peripheral blood mononuclear cells, and the development of multiple organ dysfunction in children with sepsis. Mol Med Rep 15: 2433-2442, 2017.

26. Yamazaki K, Hasegawa M, Ohoka I, Hanami K, Asoh A, Nagao T, Sugano I and Ishida Y: Increased E2F-1 expression via tumour cell proliferation and decreased apoptosis are correlated with adverse prognosis in patients with squamous cell carcinoma of the oesophagus. J Clin Pathol 58: 904-910, 2005.

27. Shou Z, Lin L, Liang J, Li JL and Chen HY: Expression and prognosis of FOXO3a and HIF-1 $\alpha$ in nasopharyngeal carcinoma. J Cancer Res Clin Oncol 138: 585-593, 2012.

28. Lu M, Zhao Y, Xu F, Wang Y, Xiang J and Chen D: The expression and prognosis of FOXO3a and Skp2 in human ovarian cancer. Med Oncol 29: 3409-3415, 2012.

29. Bradley PJ: Adenoid cystic carcinoma of the head and neck: A review. Curr Opin Otolaryngol Head Neck Surg 12: 127-132, 2004.

30. Yang X, Dai J, Li T, Zhang P, Ma Q, Li Y, Zhou J and Lei D: Expression of EMMPRIN in adenoid cystic carcinoma of salivary glands: Correlation with tumor progression and patients' prognosis. Oral Oncol 46: 755-760, 2010.

31. Zhang J, Peng B and Chen X: Expressions of nuclear factor kappaB, inducible nitric oxide synthase, and vascular endothelial growth factor in adenoid cystic carcinoma of salivary glands: Correlations with the angiogenesis and clinical outcome. Clin Cancer Res 11: 7334-7343, 2005. 